\title{
Paper Diagnostic for Direct Measurement of Fibrinogen Concentration in Whole Blood: Supplementary Section
}

\author{
Marek Bialkower ${ }^{1}$, Clare A. Manderson ${ }^{1}$, Heather McLiesh ${ }^{1}$, Rico F. Tabor ${ }^{2}$ and Gil Garnier $^{{ }^{*}}$ \\ ${ }^{1}$ BioPRIA and Department of Chemical Engineering, Monash University \\ ${ }^{2}$ School of Chemistry, Monash University, Clayton, Vic 3800, Australia \\ *For Correspondence: Gil.Garnier@,Monash.edu
}

1. Raw results after drying

\section{Carstair's Stain}

\section{REAGENTS}

i) Bouin's Fixative

(1) $250 \mathrm{~mL}$ of Formalin (37\%)

(2) $750 \mathrm{~mL}$ of saturated aqueous picric acid

(3) $50 \mathrm{~mL}$ of glacial Acetic acid

ii) Weigert's Iron Haematoxylin

(1) Solution A
(a) $5 \mathrm{~g}$ of Haematoxylin powder
(b) $500 \mathrm{~mL}$ of absolute Ethanol

(2) Solution B

(a) Add 6 grams of ferric chloride to $520 \mathrm{~mL}$ of distilled water, and then add $5 \mathrm{~mL}$ of concentrated hydrochloric acid. Stir continuously until the ferric chloride is completely dissolved.

(3) Mix solution A and B in equal parts, does not keep beyond a day

iii) Picric Acid - Orange G

(1) $200 \mathrm{ml}$ isopropanol 
(2) 2.8 g saturated picric acid

(3) $20 \mathrm{ml}$ saturated aqueous picric acid

(4) 0.2 g Orange G

iv) Ponceau-fuschin

(1) $1 \mathrm{ml}$ 100\% acetic acid

(2) $0.5 \mathrm{~g}$ acid fuschin

(3) 0.5 g Ponceau $2 R$

v) Aniline blue staining solution

(1) 25 grams of Aniline Blue

(2) $25 \mathrm{~mL}$ of glacial Acetic acid

(3) $1,000 \mathrm{~mL}$ of distilled water

vi) $1 \%$ Phosphotungstic acid

(1) 10 grams of phosphotungstic acid

(2) 1,000 mL of distilled water

\section{PROCEDURE}

1. Bring paper strips to water.

2. Place paper strips in Bouin's fixative either overnight at room temperature or 1 hour at $60^{\circ} \mathrm{C}$.

3. Wash the paper strips in running water.

4. Stain paper strips in Weigert's haematoxylin for 15 minutes.

5. Differentiate briefly in acid alcohol.

6. Wash in running tap water for 10 minutes.

7. Stain paper strips in Picric acid-orange $\mathrm{G}$ solution for 45 minutes. 
8. Wash in running tap water.

9. Stain paper strips in Ponceau-fuschin solution for 5 minutes.

10. Wash in running tap water.

11. Apply $1 \%$ tungstophosphoric acid for 15 minutes (not required to wash sections after this step).

12. Stain with Aniline blue staining solution for 20 minutes.

13. Wash in running tap water.

14. Dehydrate through 3 changes of ethanol.

15. Clear in 3 changes of xylene.

16. Mount with DPX. 


\section{Paper strip Cut out:}

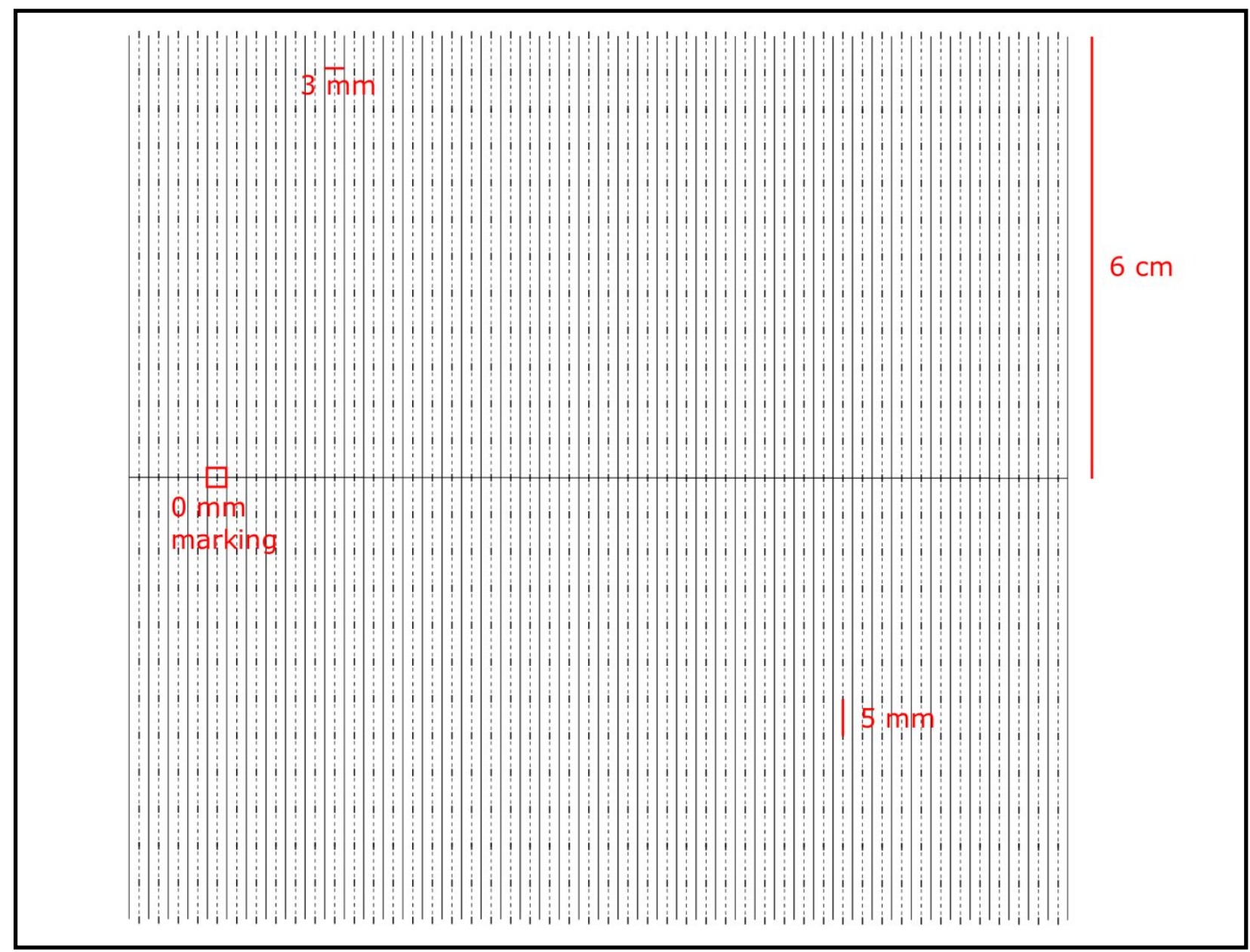

Supplementary Figure 1: Cut out of Paper Strips showing dimensions, markings and locations. The paper strip cutout stencil was drawn on CoreIDRAW X6 and a 60 Watt Epilog Helix was used to laser cut the paper.

After laser cutting the paper with the stencil used in Supplementary Figure 1, paper strips were ripped out individually with tweezers and experimented with.

The $0 \mathrm{~mm}$ marking refers to the location where the paper strip was dropped on top of the clotted droplet. The $0 \mathrm{~mm}$ marking aligned with the edge of the clotted droplet covering it in whole. 


\section{Manual Camera Setup}

In order to capture videos which manually focused on the strip's markings, ProMovie was used on an iPhone 6. The zoom was adjusted to $3.00 \mathrm{X}$ to get maximum zoomage and the focus was changed until the markings on the paper strips could be clearly seen (as shown in Supplementary Figure 2).

The videos recorded could then be screenshotted and used for analysis on ImageJ.

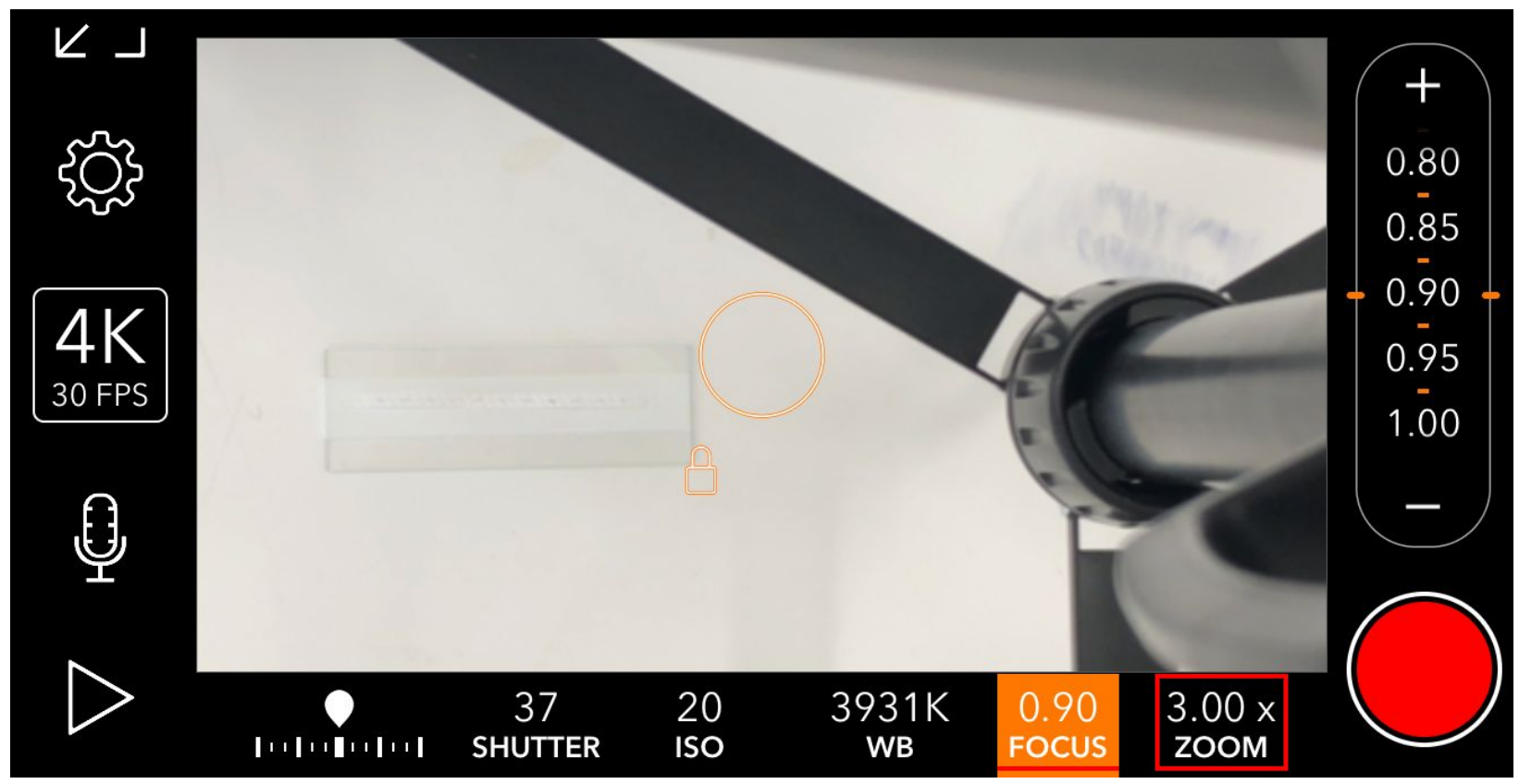

Supplementary Figure 2: Settings on ProMovie used to manually focus and zoom on the paper strip's markings. 
4. Measuring Process:

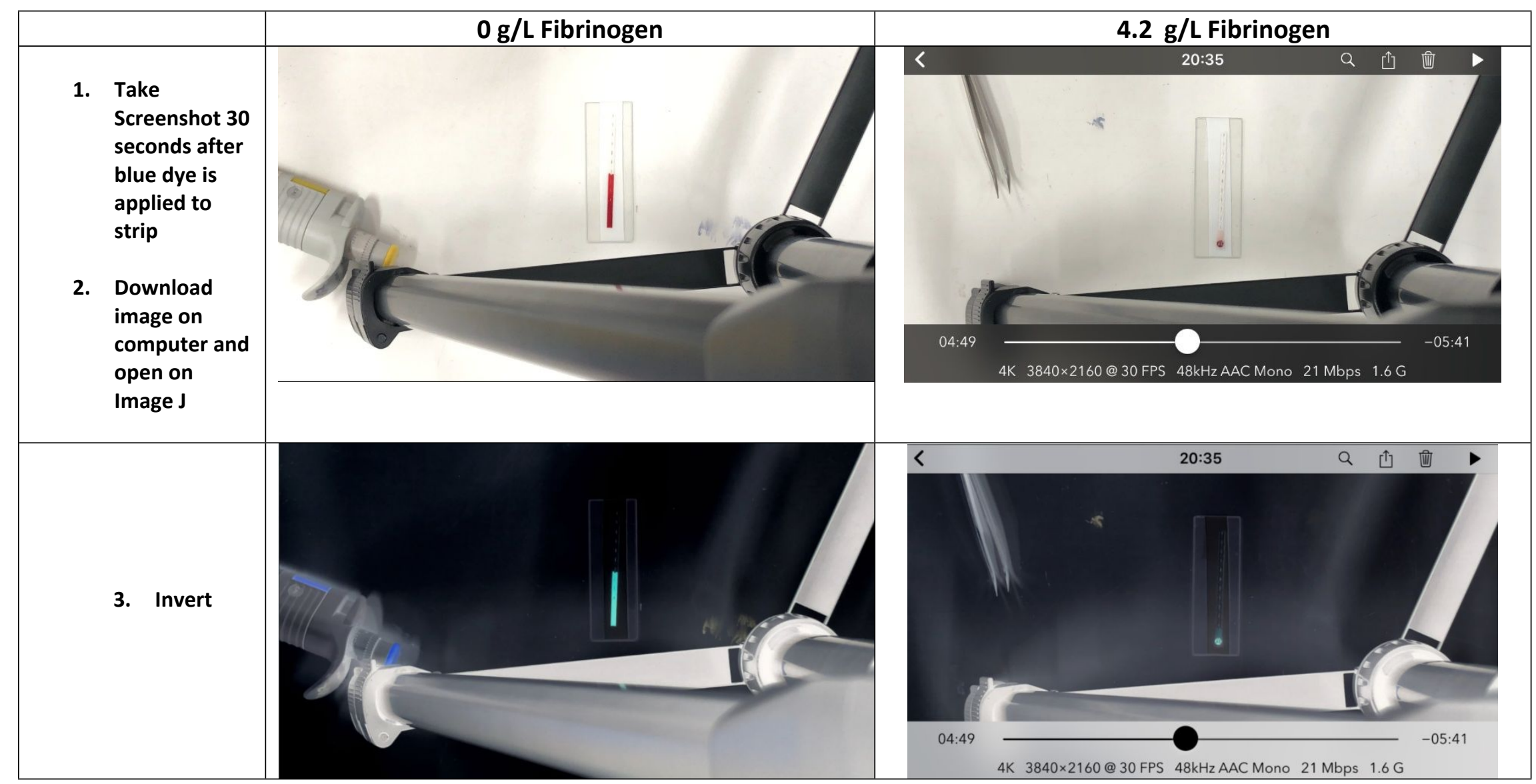




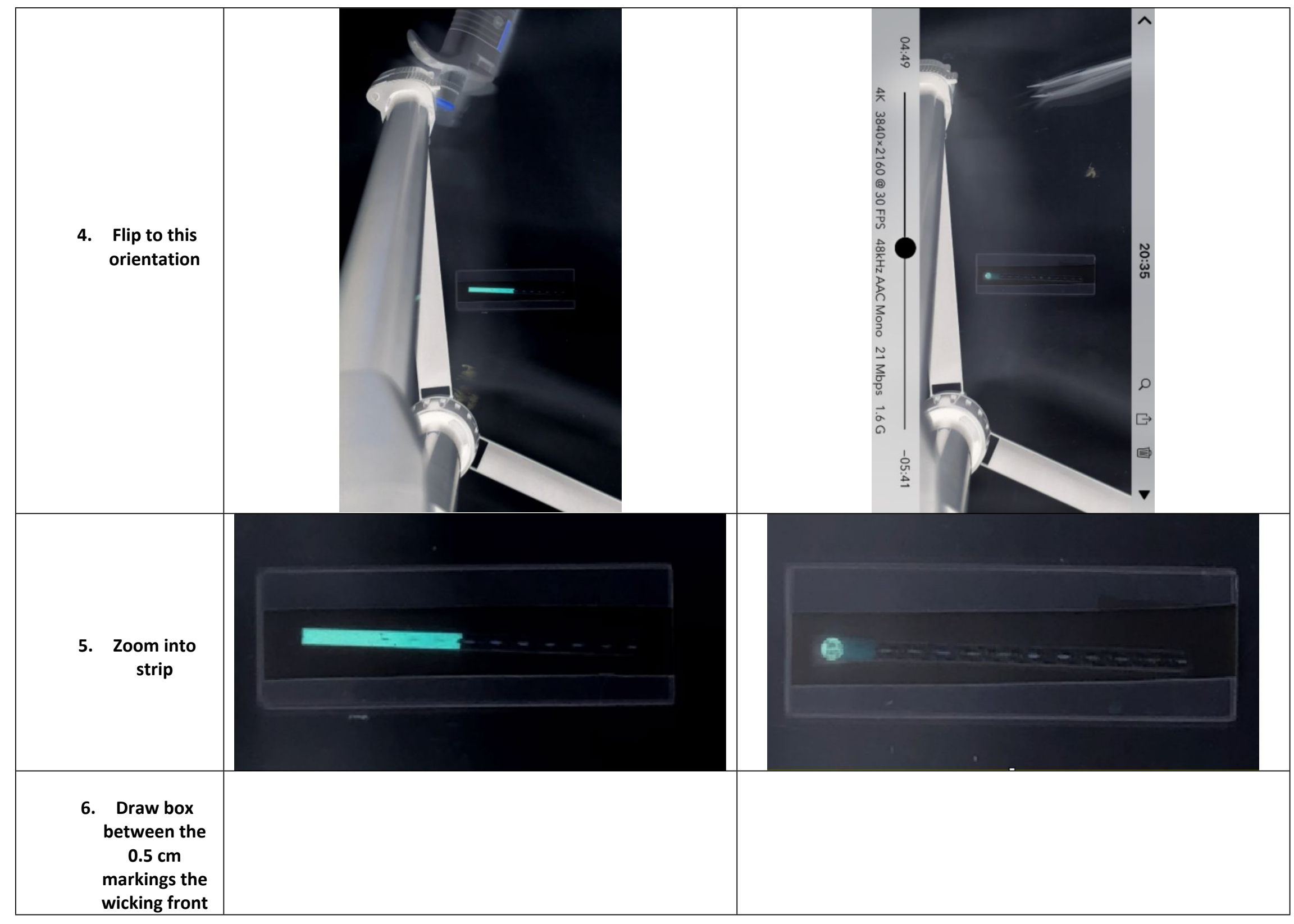




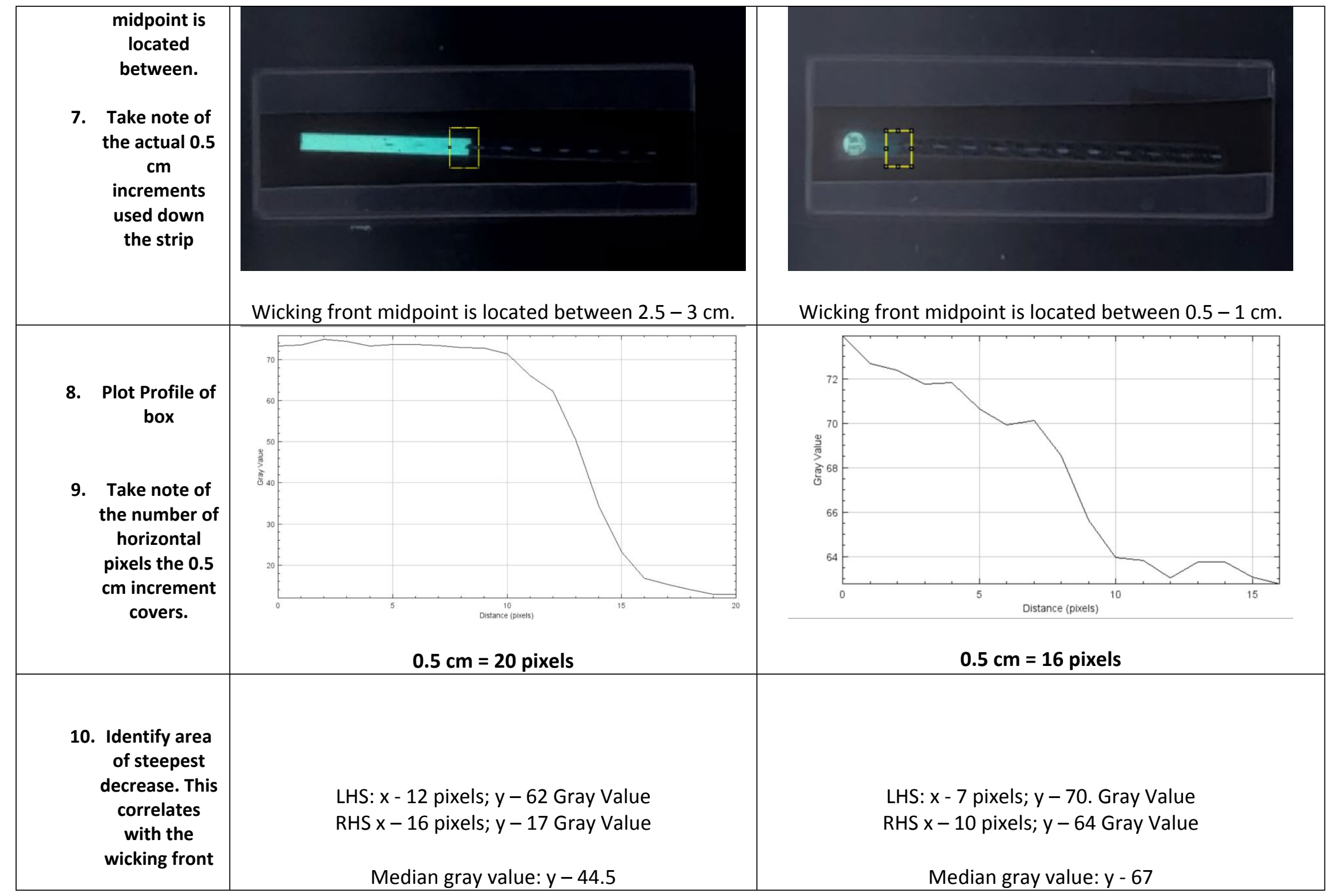




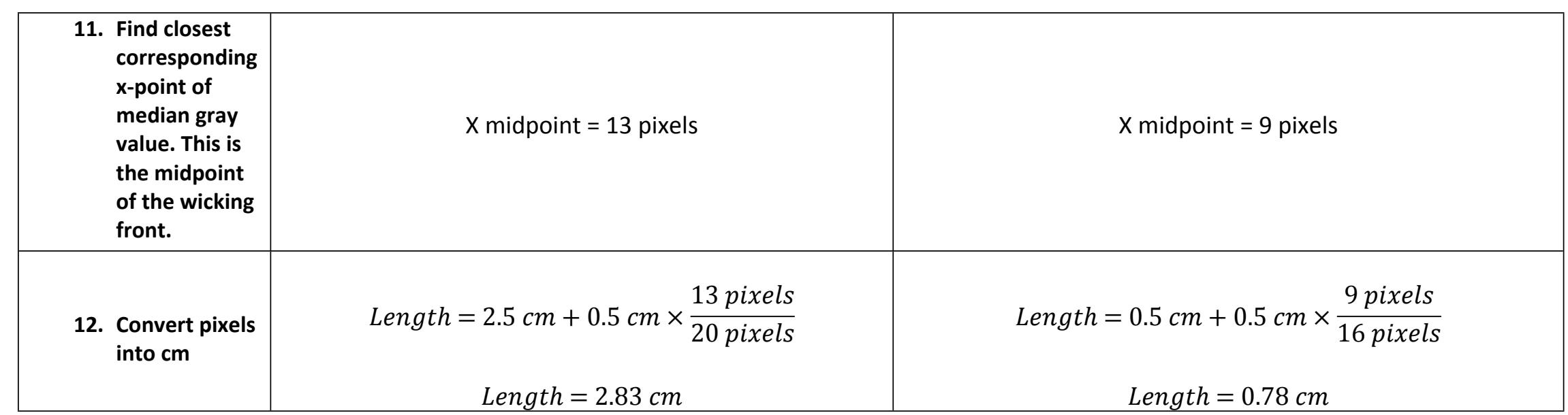

Supplementary Figure 3: Step by step process of calculating wicking length on Image J. 


\section{Effect of Humidity}

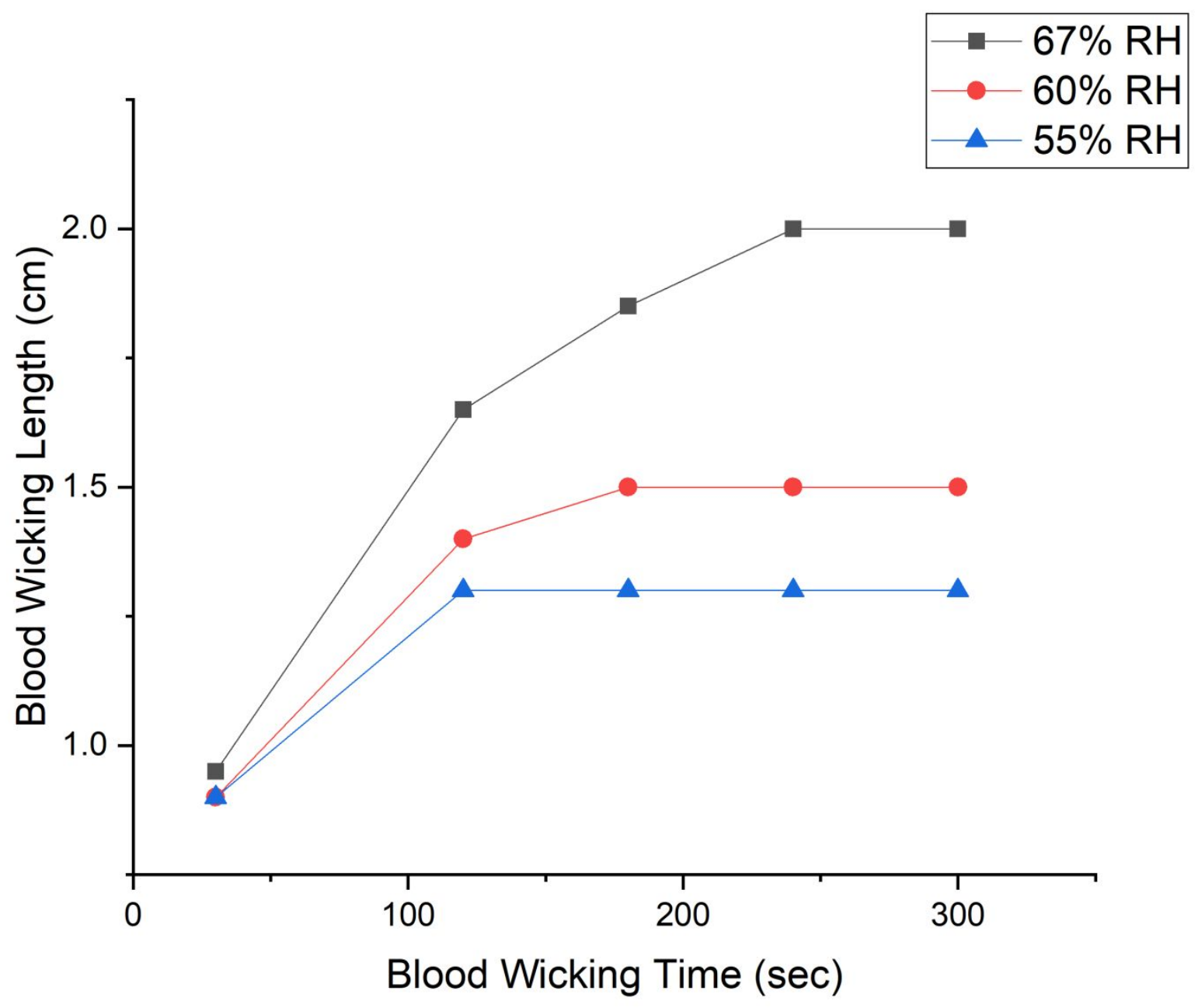

Supplementary Figure 4: Testing of $3.2 \mathrm{~g} / \mathrm{L}$ whole blood sample at different relative humidities. Whole blood reacted with thrombin for 3 minutes and blood wicking length was measured using ImageJ. Each data point was tested 1 time.

Supplementary Figure 4 demonstrates a single whole blood sample wicking at different relative humidities to observe the effects of drying on wicking blood. At smaller wicking times, the blood wicking lengths are quite similar. However, at longer wicking times the effect of drying becomes more pronounced. Whole blood droplets at low relative humidities dry out faster than at high relative humidities. Hence, they stop wicking at both shorter wicking times and lengths. Therefore, to minimise the effects of humidity, the test is more suitable to wicking for short times (ie. 30 seconds) than long times. 


\section{Raw results after drying}

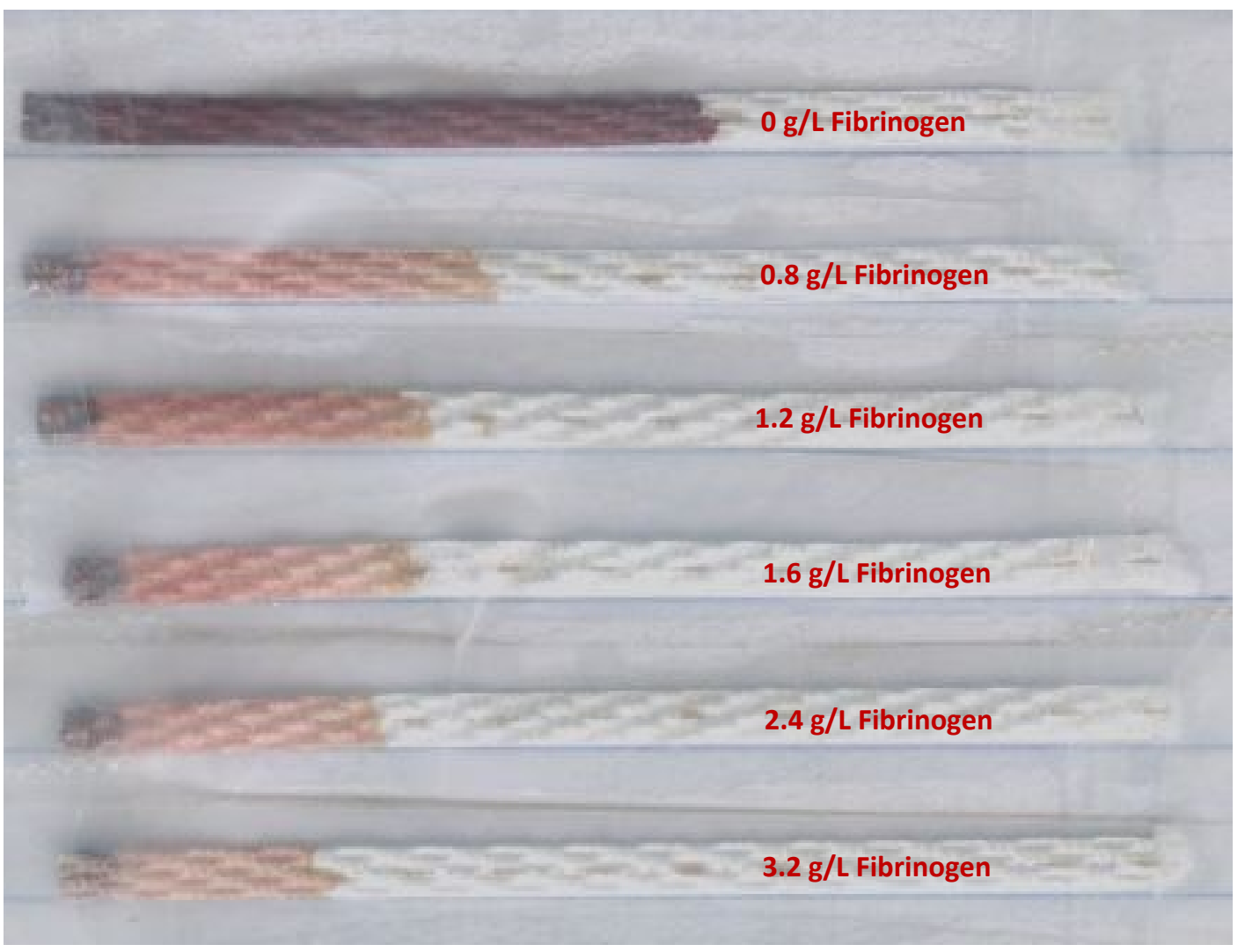

Supplementary Figure 5: Raw results from Figure 3. Whole blood samples of different fibrinogen concentrations were tested. Paper strips were collected after drying and scanned. Lower fibrinogen concentrations causes further blood wicking to occur.

\section{Effect of Reaction Time}

Supplementary Figure 6 investigates the effect of reaction time to observe how long it takes for the reaction to complete. Whole blood droplets reacted with thrombin for different periods of time before a paper strip was placed on top of them. The longer the reaction time the greater the wicking length is perturbed. At 30 seconds, a plasma fibrinogen concentration vs blood wicking length curve is already developing. However, it is not developed until at least 120 seconds. At 180 seconds, no more significant change occurs in the curve. This indicates that the reaction is completed in between 120 seconds and 180 seconds. 


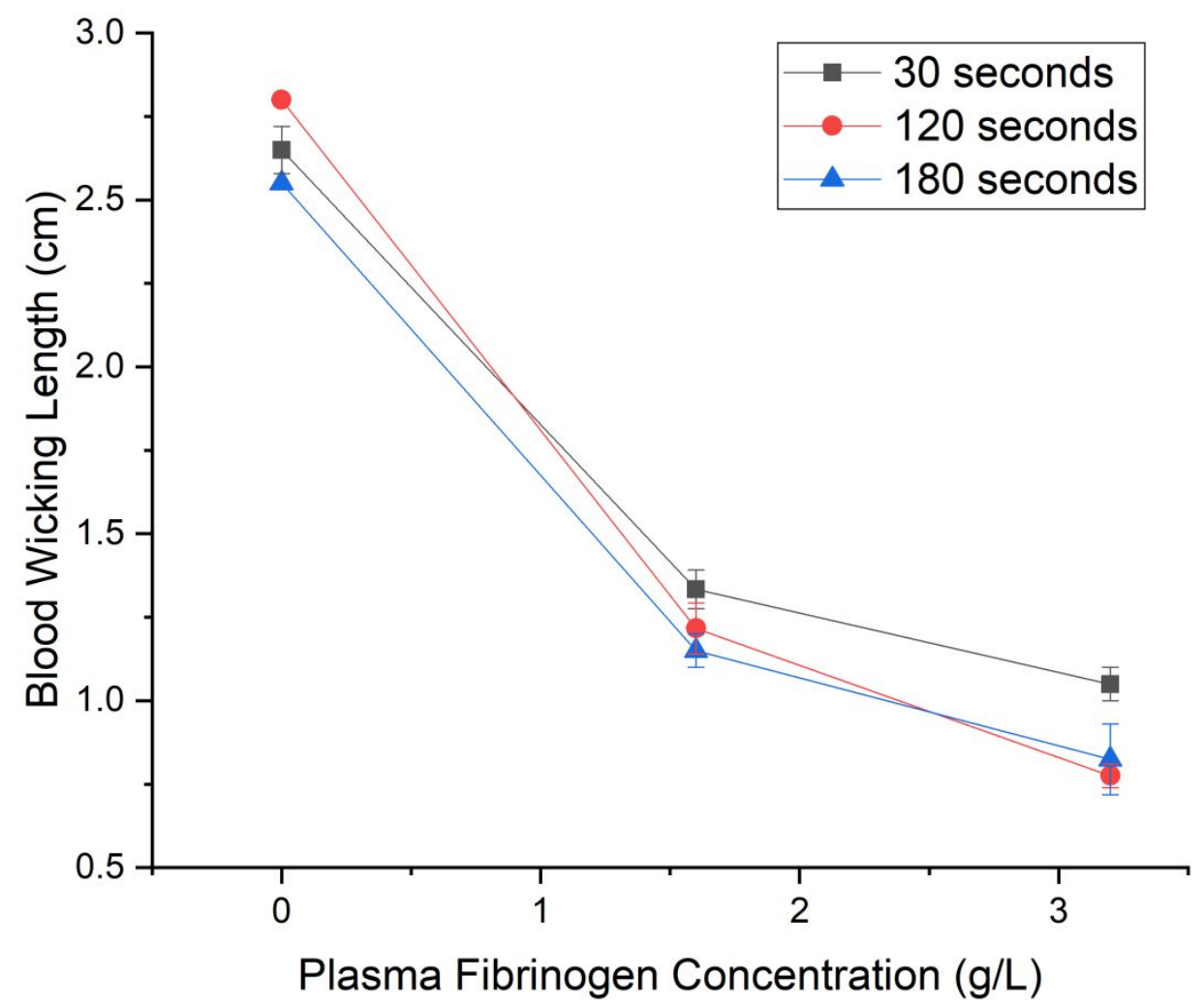

Supplementary Figure 6: Testing of whole blood samples under different reaction times. Blood wicking length was measured after 30 seconds of wicking using ImageJ. Each data point was tested 3 times and the mean and standard deviation are reported. 


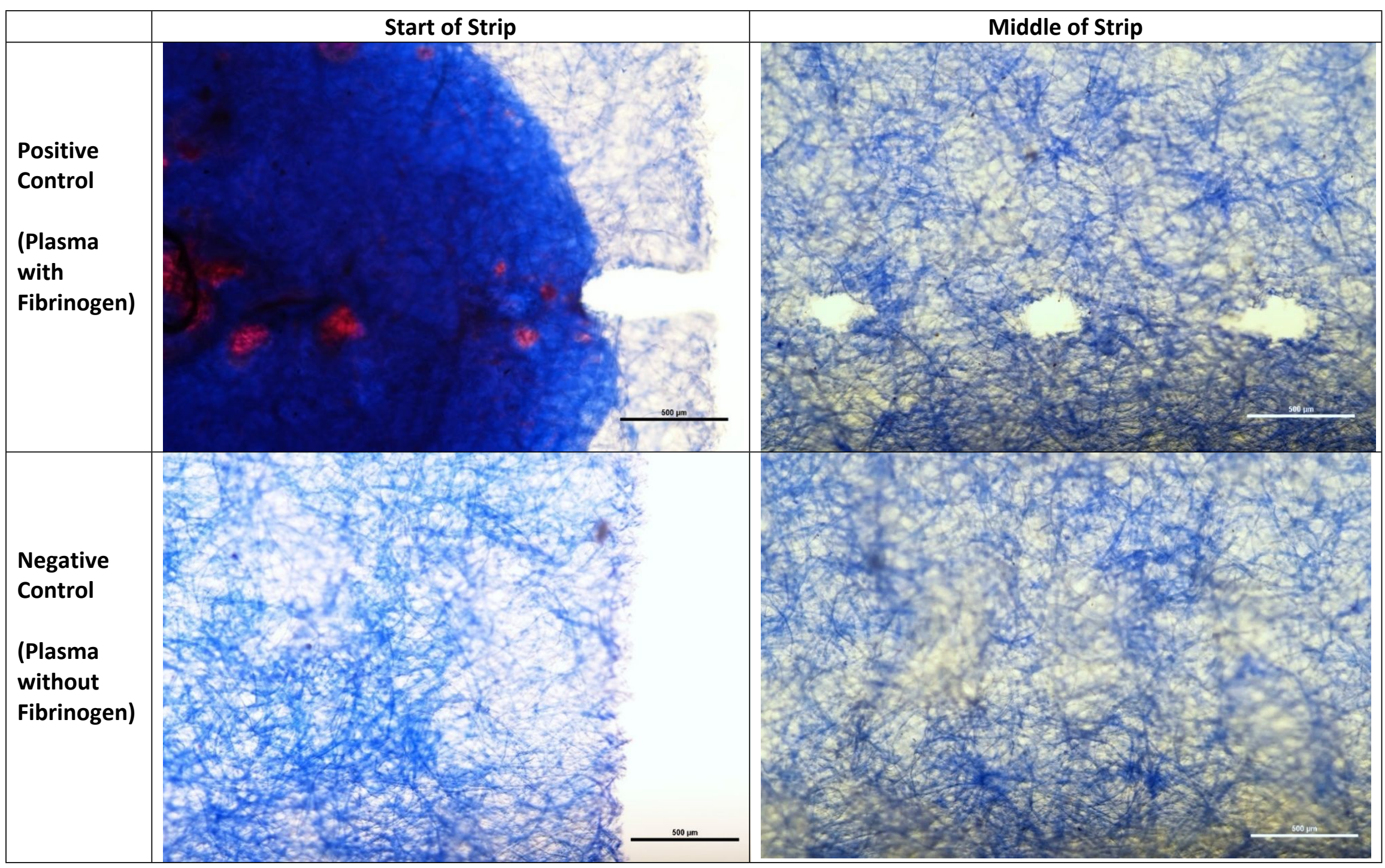

Supplementary Figure 7: Histological staining and imaging of plasma samples on paper with (positive control) and without (negative control) fibrinogen. Plasma droplets were deposited on a PTFE coated glass slide, mixed with thrombin and reacted for 3 minutes, before a paper strip was placed on top of the droplet. After drying, the paper strips underwent a Carstairs stain to visualise the location of fibrin throughout the paper. Imaging was performed on a Nikon Eclipse Ni-E Upright Microscope at (5X zoom). Red = Fibrin; Blue = Cellulose. 


\section{Fibrin Staining}

Supplementary Figure 7 illustrates the location of fibrin along the strip after drying to determine whether the fibrin moves with the wicking fluid as one phase or remains in the droplet during wicking. Both a positive control (plasma with fibrinogen) and a negative control (plasma without fibrinogen) were imaged. A Carstair's stain was performed to visualise the location of fibrin along the paper strip (See Supplementary Section 1). Fibrin deposits are coloured red, cellulose fibres are coloured blue. The start of the strip refers to the position where the paper strip was placed on top of the clotted droplet. The middle of the strip refers to a location approximately $3 \mathrm{~cm}$ down the strip away from the clotted droplet - where blood has clearly wicked to. There is a strong presence of fibrin deposits at the start of the strip for the positive control, but not the negative control. However, there is no presence of fibrin in the middle of either strip. This means that the fibrin remains in the clotted droplet and does not wick down the strip. Only the remaining serum fluid from the clotted droplet wicks down the strip. Therefore, once the droplet has reacted with thrombin, 2 phases are formed: a clotted phase and an unclotted phase. When the strip is placed on the clotted droplet, only the unclotted phase can wick down the strip. 


\section{Effect of Droplet Contact Angle}

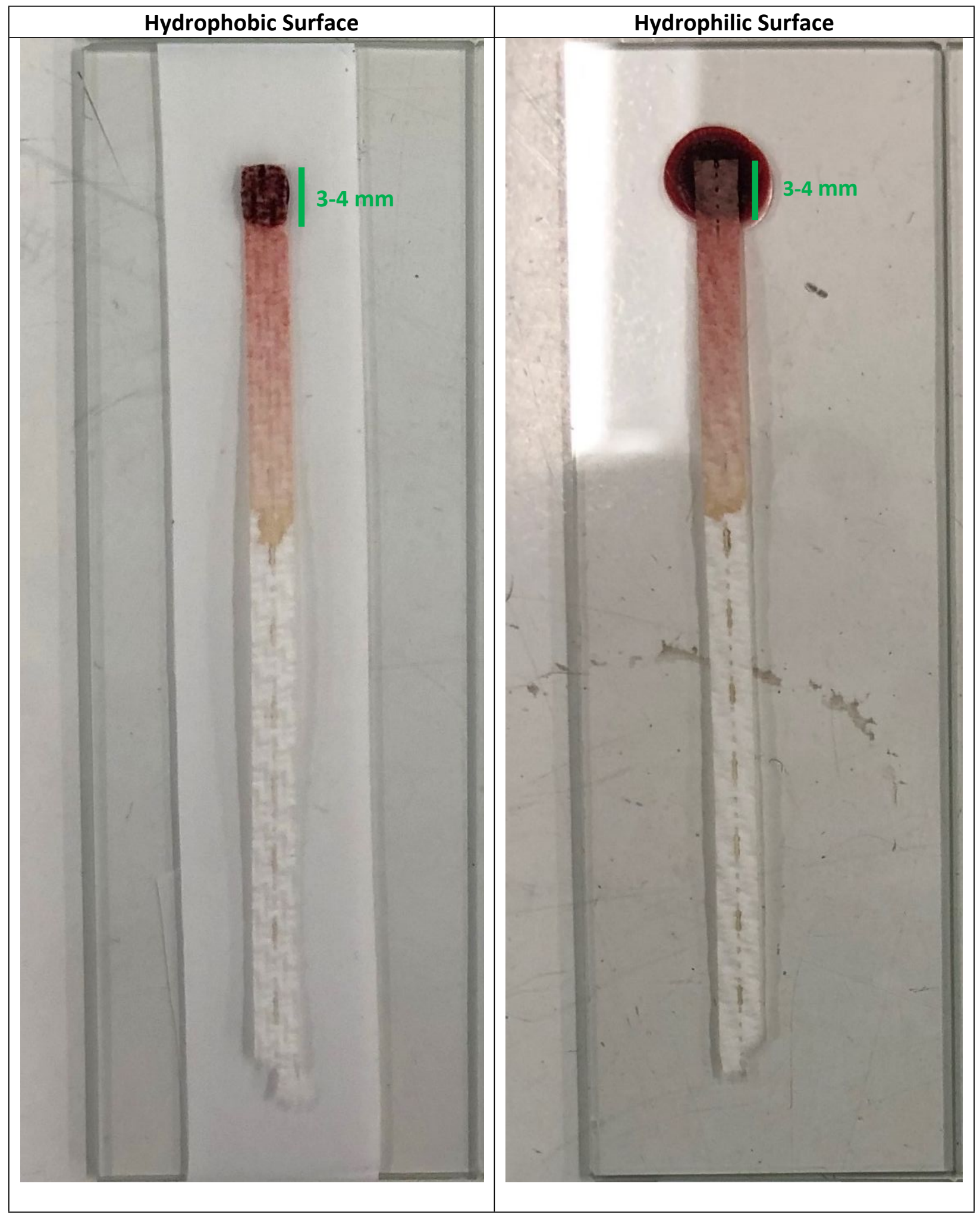

Supplementary Figure 8: Comparison of the test performed on hydrophobic and hydrophilic-surfaced glass slides after it has completely dried and wicked no more. 
Supplementary Figure 8 compares the wicking of blood on hydrophobic surfaces and hydrophilic surfaces. Since hydrophilic surfaces produce droplets with lower contact angles, it also causes the droplet to spread out much further. To ensure the comparison was controlled, the contact area between the paper strip and the clotted droplet was kept consistent. This was achieved by allowing an interfacial contact length between 3-4 $\mathrm{mm}$ to form.

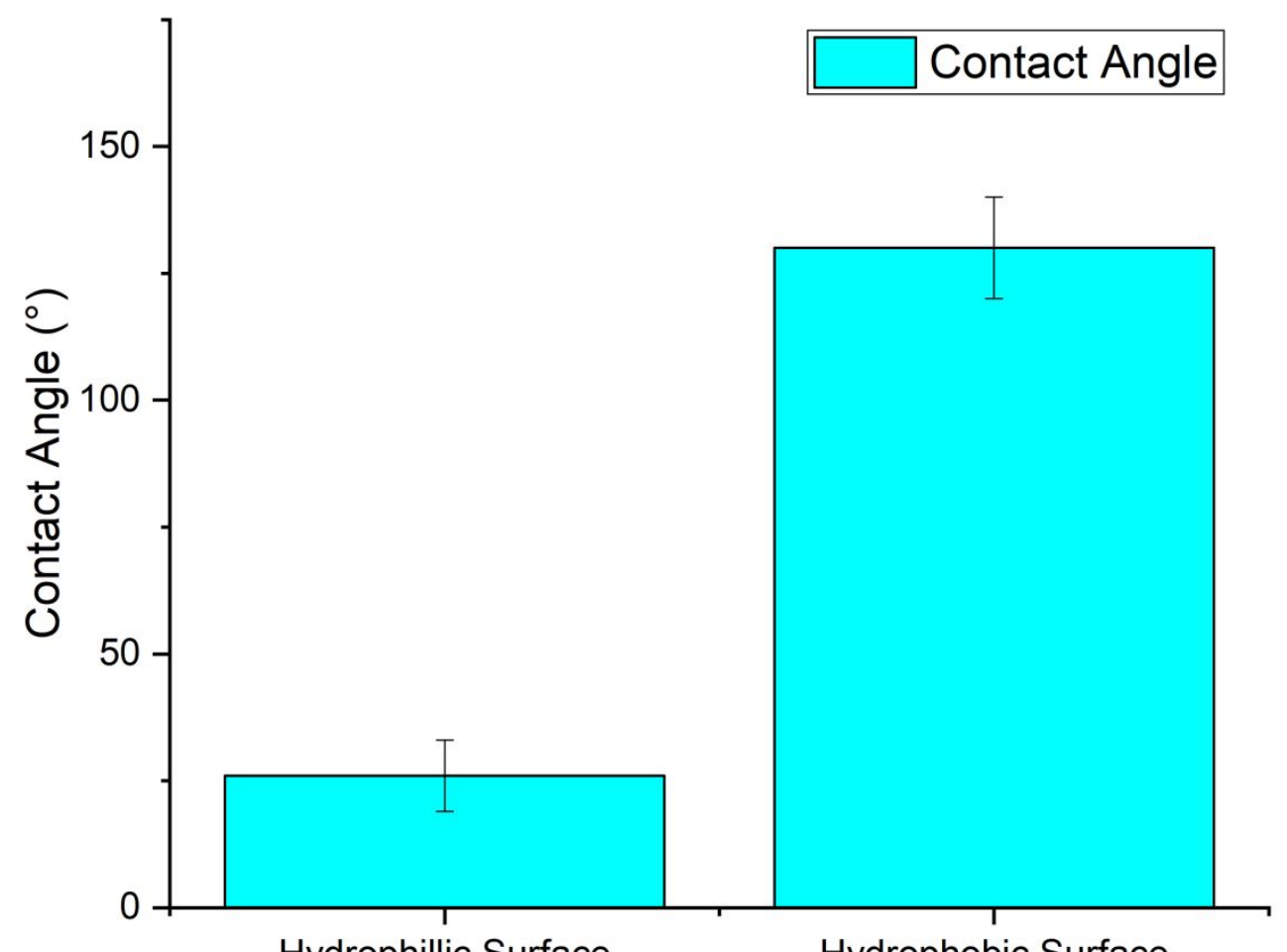

Hydrophillic Surface

Hydrophobic Surface

Supplementary Figure 9: Measurement of water droplet contact angle on hydrophobic and hydrophilic-surfaced glass slides. Each data point was tested 3 times and the mean and standard deviation are reported.

Supplementary Figure 9 determines the water droplet contact angle of both the hydrophobic and hydrophilic-surfaced glass slides. The contact angle was measured using a Dataphysics OCA35 device. A $2 \mu \mathrm{L}$ droplet of water was deposited onto the surface and the contact angle was measured afterwards. The difference in contact angle is significant. The hydrophilic surface produced a contact angle averaging $25^{\circ}$ whilst the hydrophobic surface produced a contact angle averaging $130^{\circ}$. Respectively, these angles are indicative of the angles of the unbeaded and beaded droplets formed in the actual test. 

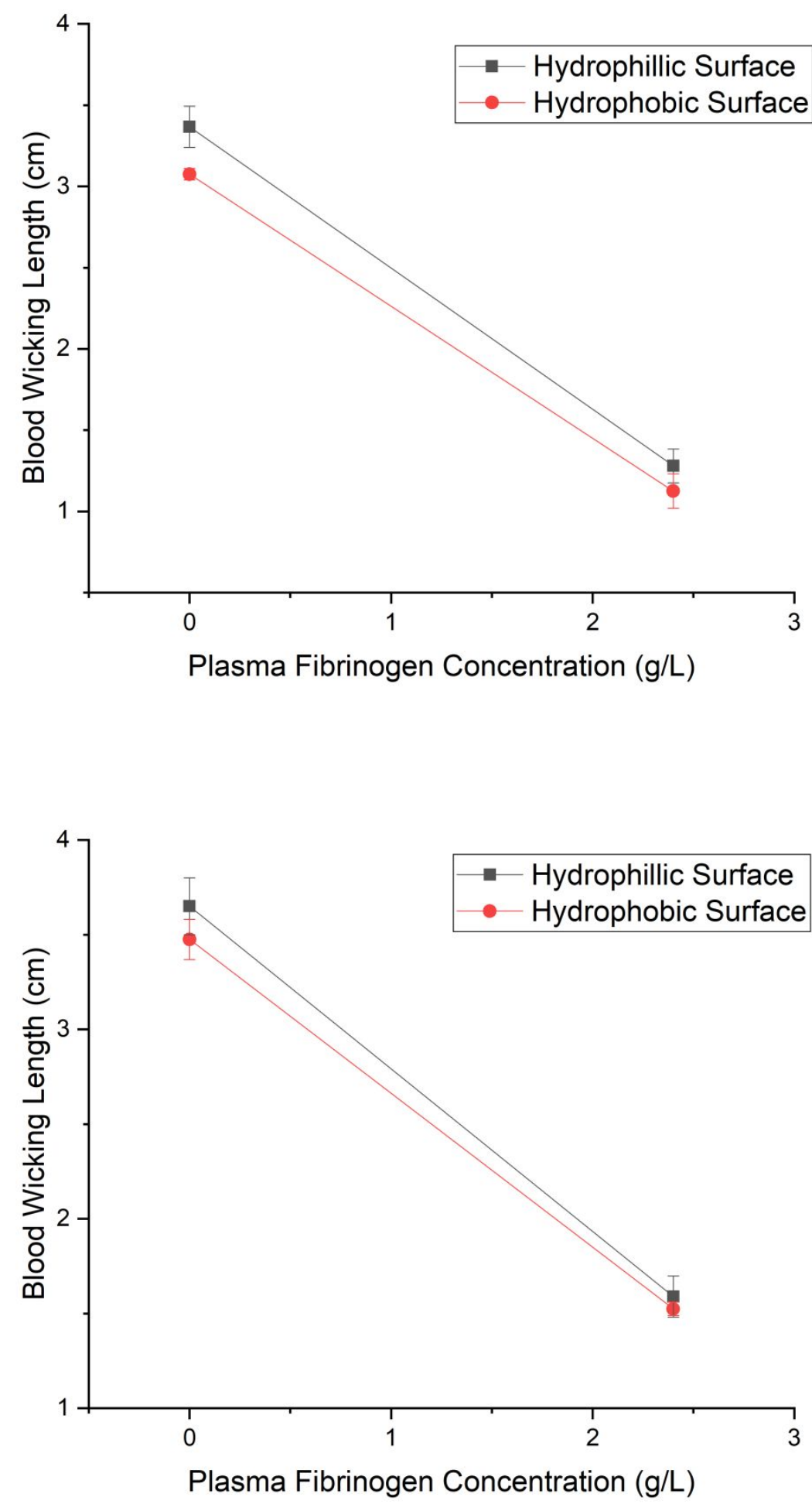

Supplementary Figure 10: Testing of hydrophobic and hydrophilic-surfaced glass slides. Whole blood reacted with thrombin for 3 minutes and the blood wicking length was measured after 30 seconds (top) and 60 seconds (bottom) of wicking using ImageJ. Each data point was tested 3 times and the mean and standard deviation are reported. 
Supplementary Figure 10 investigates the effects of droplet contact angle and measures the blood wicking length after different wicking times. Unbeaded droplets and beaded droplets are tested on hydrophobic and hydrophilic surfaces, respectively. After 30 seconds (top graph) there are insignificant differences in wicking. Unbeaded droplets wick slightly further than beaded droplets. This is hypothesised to be due to the unbeaded droplets ability to wet the paper more efficiently. However, no difference in sensitivity is observed. After 60 seconds, the initial wetting effect becomes neutralised. Both unbeaded and beaded droplets wick to the same lengths. Despite the significantly different contact angle unbeaded and beaded droplets display in Supplementary Figure 9, the overall effect it has on the test is subtle. Hence, the test can work with different solid surface materials and droplet contact angles. 
10. Relationship between haematocrit, viscosity and blood wicking length
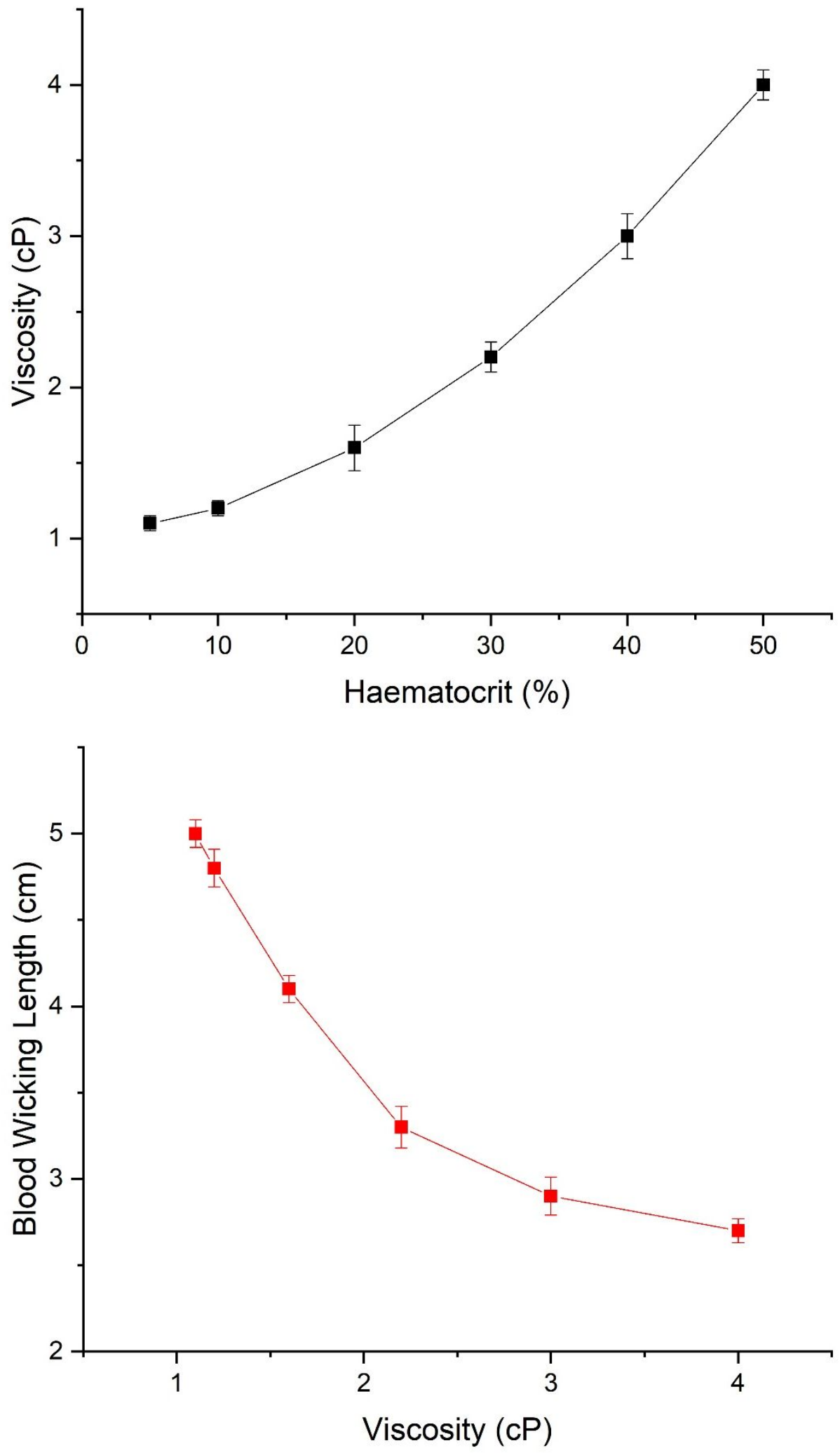


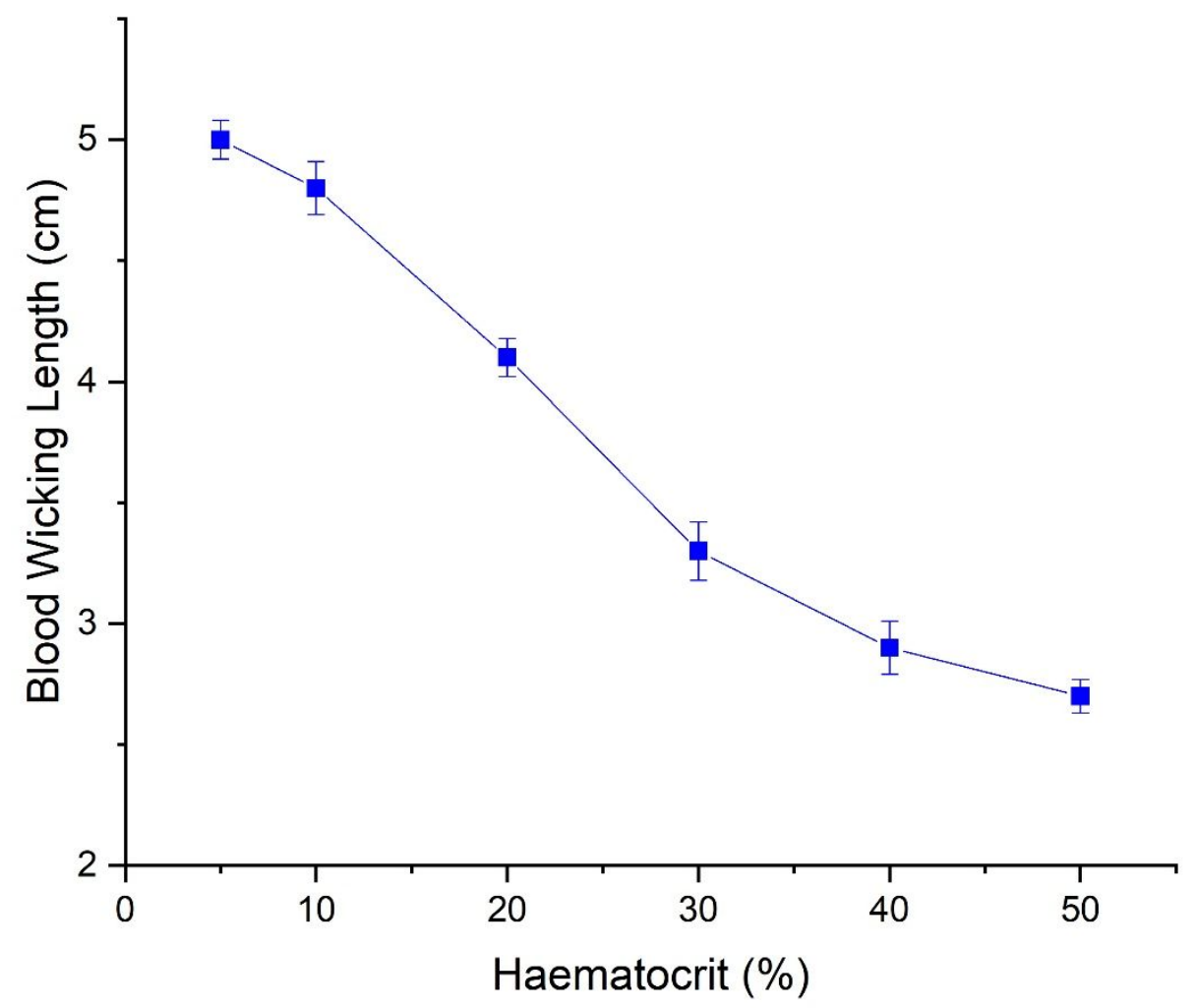

Supplementary Figure 11: The relationship between haematocrit, viscosity and blood wicking length deciphered from artificially prepared blood samples. Top Graph: haematocrit vs viscosity. Middle graph: viscosity vs blood wicking length. Bottom graph: haematocrit vs blood wicking length. Each data point was tested 3 times and the mean and standard deviation are reported.

Supplementary Figure 11 determines how haematocrit, viscosity and blood wicking length all relate to each other. Blood samples were prepared by washing red blood cells ( as described in the methodology section) and diluted in PBS to different haematocrits. The viscosity of each blood sample was measured using a Cannon-Fenske viscometer of size 200 ( $0.1 \mathrm{cSt} / \mathrm{s})$. Since the density of whole blood (ie. $1.06 \mathrm{~g} / \mathrm{mL}$ ) is very similar to that of PBS solution (ie. $1.00 \mathrm{~g} / \mathrm{mL}$ ), the density used to convert cSt to $\mathrm{CP}$ was assumed to be $1.00 \mathrm{~g} / \mathrm{mL}$ for all blood samples. The blood wicking length was measured using the main test procedure (described in the methodology section).

The top graph shows an exponentially increasing relationship between haematocrit and viscosity. Haematocrits above $10 \%$ show significant changes in viscosity. However, haematocrits below this value do not change significantly.

The middle graph shows an exponentially decaying relationship between viscosity and blood wicking length. The effect of viscosity changes significantly up until $3 \mathrm{cP}$. After that, the effect of viscosity becomes insignificant. 
The bottom graph shows a sigmoidal relationship between haematocrit and blood wicking length. This shape arises due to: 1 ) the insignificant changes in viscosity below $10 \%$ haematocrit (as shown in the top graph); 2) the insignificant effect of viscosity on blood wicking length above $40 \%$ haematocrit (as shown in the middle graph). 


\section{Relationship between Droplet Fibrinogen Concentration and Contact Area}

\begin{tabular}{|c|l|}
\hline Fibrinogen Concentration & \multicolumn{1}{c|}{ Fibrinogen Concentration } \\
\hline $0.4 \mathrm{~g} / \mathrm{L}$ Fibrinogen & $\bar{\approx} \mathrm{mm}$ \\
\hline $0.2 \mathrm{~g} / \mathrm{L}$ Fibrinogen & $\bar{\approx}$ \\
& $\approx 4.5 \mathrm{~mm}$ \\
$0.1 \mathrm{~g} / \mathrm{L}$ Fibrinogen & $\overline{\approx 6 \mathrm{~mm}}$ \\
\hline
\end{tabular}

Supplementary Figure 12: Comparison of the contact length between the paper strip and clotted droplets of different fibrinogen concentrations. Images were taken after they had completely dried and wicked no more.

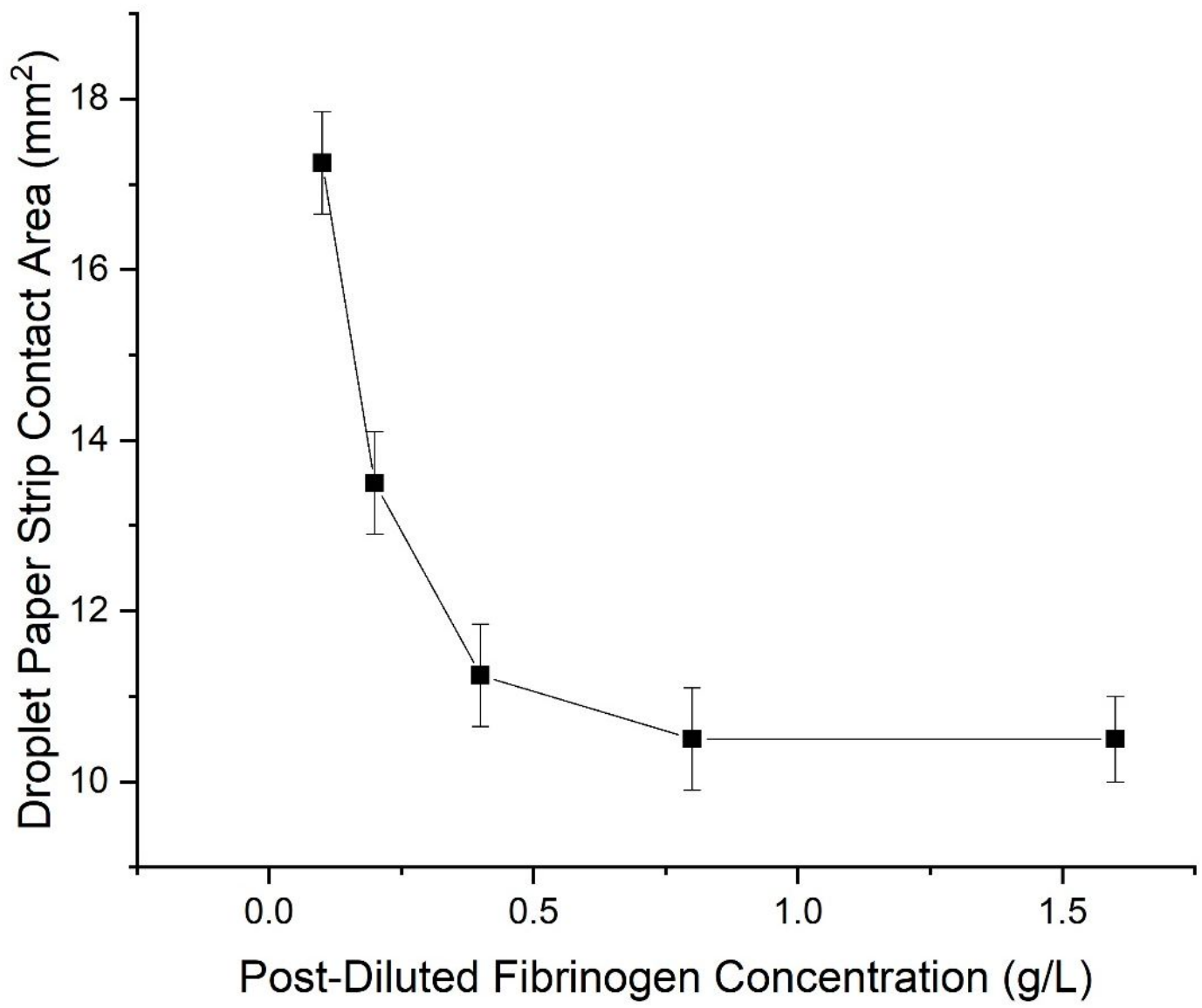

Supplementary Figure 13: The relationship between the clotted droplet fibrinogen concentration and the contact area with the paper strip. Each data point was tested 4 times and the mean and standard deviation are reported. 
Supplementary Figure 12 and $\mathbf{1 3}$ explores the relationship between the clotted droplet fibrinogen concentration and the contact area with the paper strip. This in turn explains how the droplet stiffness impacts on blood wicking length and why Graph 4 linearises at higher dilutions.

As the clotted droplet's fibrinogen concentration decreases, so does its stiffness (as less fibrin is present). At fibrinogen concentrations below $0.4 \mathrm{~g} / \mathrm{L}$, the stiffness drops so low that it compresses underneath the weight of the paper. As the fibrinogen concentration drops, the compression increases exponentially. Consequentially, the contact area between the paper strip and the clotted droplet also increases. This causes more blood to wick out of the droplet than it would have otherwise if the clotted droplet resisted compression.

This can be explained with Darcy's Law:

$$
q=-\frac{k \Delta P}{\mu L}
$$

And the volumetric flux equation:

$$
q=\frac{L_{2} A_{2}}{t A_{1}}
$$

Where $L_{2}$ is the blood wicking length, $A_{2}$ is the paper cross sectional area, $t$ is the wicking time and $A_{1}$ is the contact area between the clotted droplet and the paper strip.

From Supplementary Figure 13, the clotted droplet resists compression when its fibrinogen concentration ranges from 0.4-1.6 g/L. This is relevant for the curves created for the 1:0 and 1:1 dilutions.

The only factor modulating the blood wicking length between these concentrations is the permeability of the droplet $(k)$. Hence, the permeability of the droplet is proportional to the blood wicking length:

$$
k \propto L_{2} \text { (3) }
$$

Given that higher fibrinogen concentrations ${ }_{c}$ ) create droplets with lower permeabilities, the 2 parameters are inversely proportional to each other. Hence the blood wicking length will also be inversely proportional to the fibrinogen concentration:

$$
c^{n} \propto \frac{1}{k}
$$

Hence the fibrinogen concentration vs blood wicking length relationship seen for these concentrations is non-linear.

At larger dilutions (ie. 1:3 and 1:7), the curve linearises. These dilutions correspond to clotted droplet fibrinogen concentrations below $0.4 \mathrm{~g} / \mathrm{L}$. As the fibrinogen concentration drops below this range, the blood wicking length becomes modulated by both the clot permeability and 
contact area. Each time the clotted droplet's fibrinogen concentration halves, the contact area increases by roughly $25 \%$. Hence, it wicks $25 \%$ further than it would on permeability alone. This increase is enough to convert the non-linear relationship seen with the 1:1 dilution curve to a linear relationship seen with the 1:3 dilution curve. 


\section{Relationship between Fibrinogen Concentration and Clotting Time}

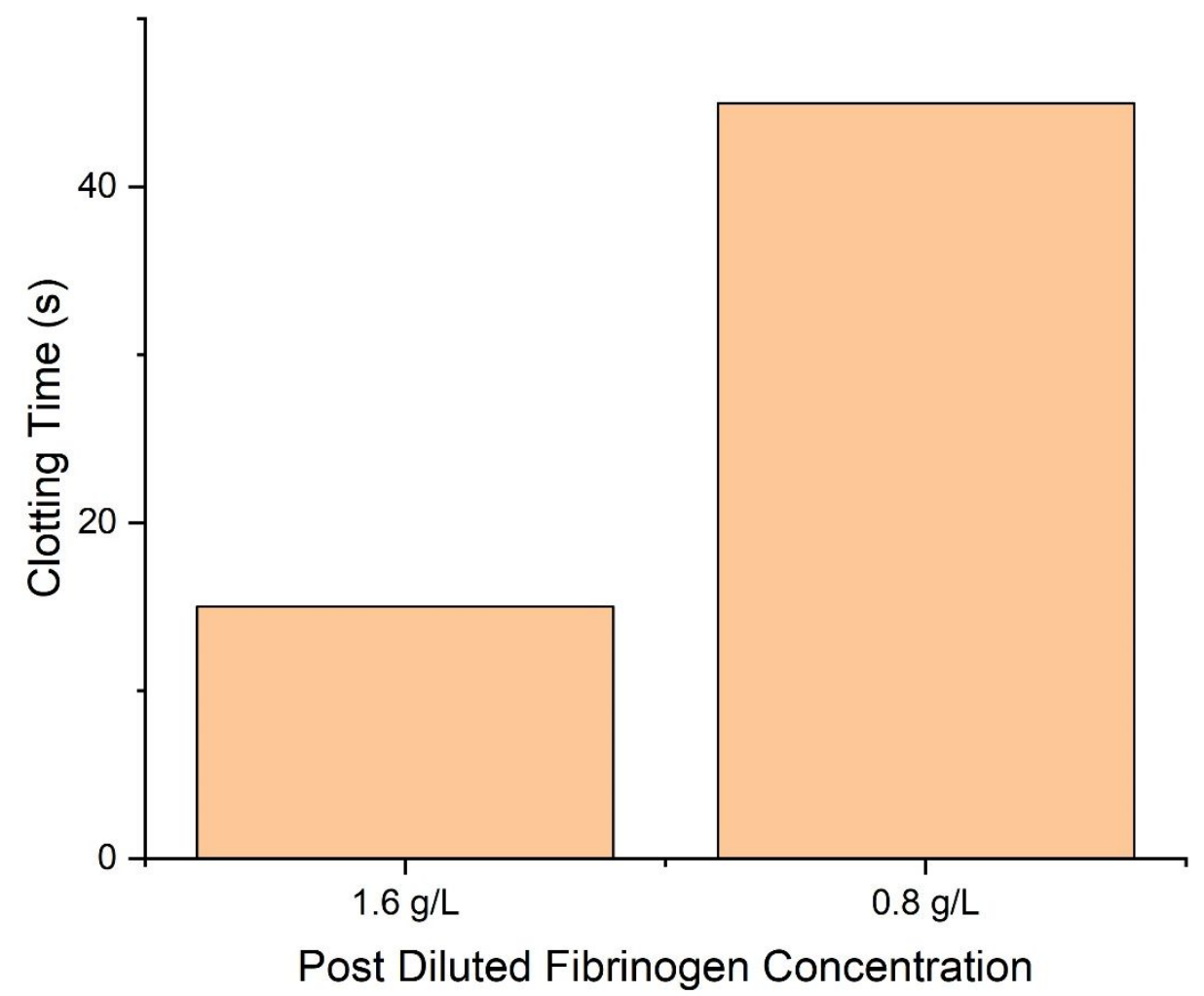

Supplementary Figure 14: The relationship between the droplet fibrinogen concentration and the time before it clots. The procedure for determining the clotting time is given in Supplementary Figure 15. The clotting time for each data point was verified 3 times.

Supplementary Figure 14 demonstrates the time taken to clot a droplet after it has been first mixed with the thrombin solution. The longer the clotting time the better, as more time is afforded for the end-user to be able to successfully mix and deposit the droplet onto the glass slide. A whole blood sample was prepared from normal plasma (as described in Section $\mathbf{3 . 3}$ of the methodology). This sample was then serially diluted with PBS (as described in Section 3.9) to produce other samples of lower fibrinogen concentrations.

To measure clotting time, $5 \mu \mathrm{L}$ of thrombin was added to a PCR tube. It was then mixed repetitively with a pipette containing $25 \mu \mathrm{L}$ of blood. The timing started the moment the blood and thrombin solution first mixed. Once the blood and thrombin solution mixture appeared uniform, it was re-suctioned into the pipette so that the clotting time could be determined. The clotting time was determined as the time the mixture could no longer escape from the pipette tip. The formation of the clot prevented the mixture from being dispensable from the tip. The clotting time was calculated by iteration using the procedure given in Supplementary Figure 15: 


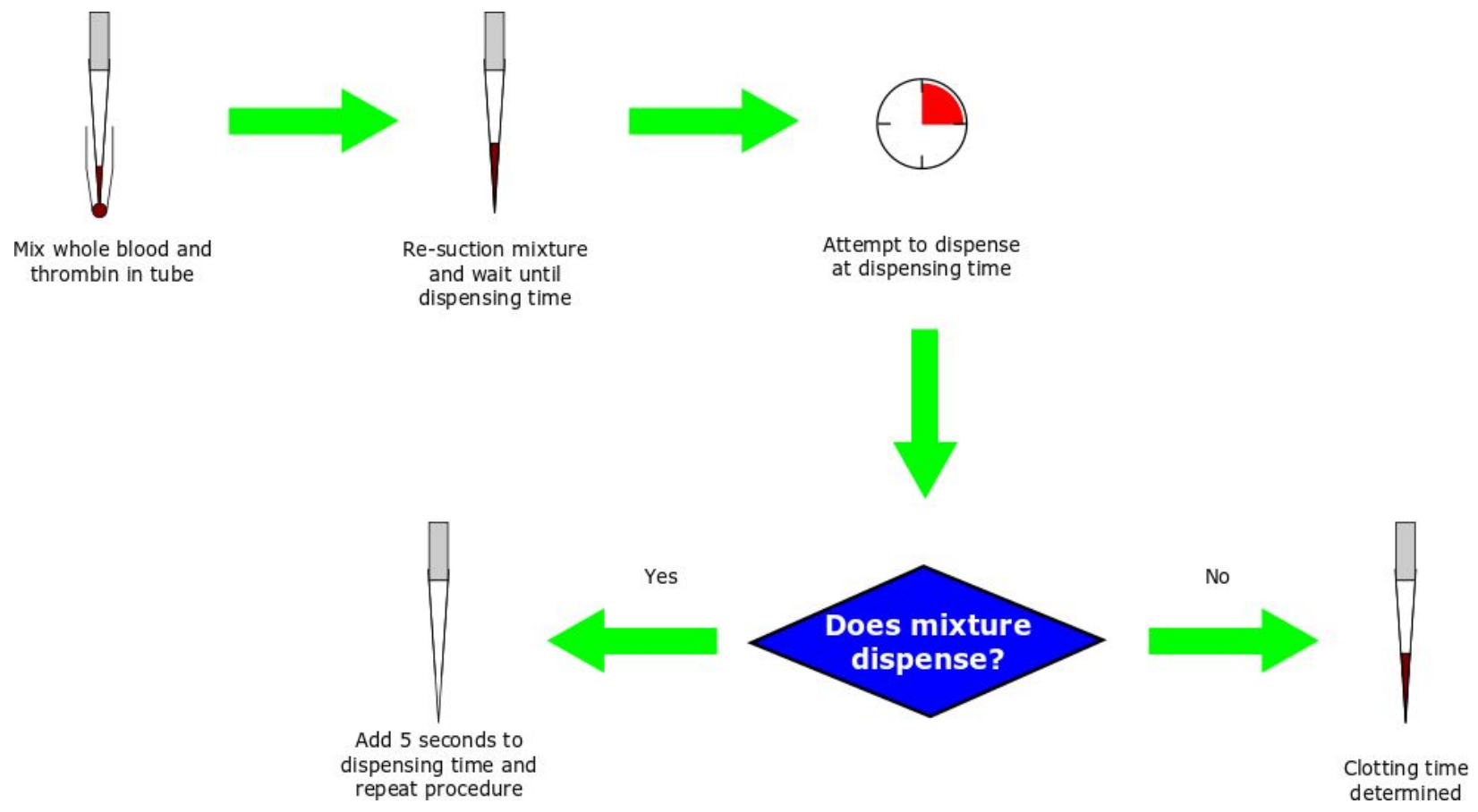

Supplementary Figure 15: Summary of methodology to determine the clotting time for droplets of different fibrinogen concentrations. The clotting time was determined as the time the mixture could no longer be dispensed from the pipette. Dispensing times began 10 seconds after the starting time and determined iteratively. 5 seconds was added to each iteration until clotting was evident.

The clotting time increases with decreasing fibrinogen concentration. This is a well-known phenomenon seen in Clauss assays. An undiluted whole blood sample of normal fibrinogen concentration clots after 15 seconds. However, diluting by half, causes the clotting time to increase to 45 seconds. Hence, by diluting, the time afforded to the end user before clotting increases. This makes the test easier to perform. 


\section{Effect of Paper Strip Dropping Height in Prototype Diagnostic}

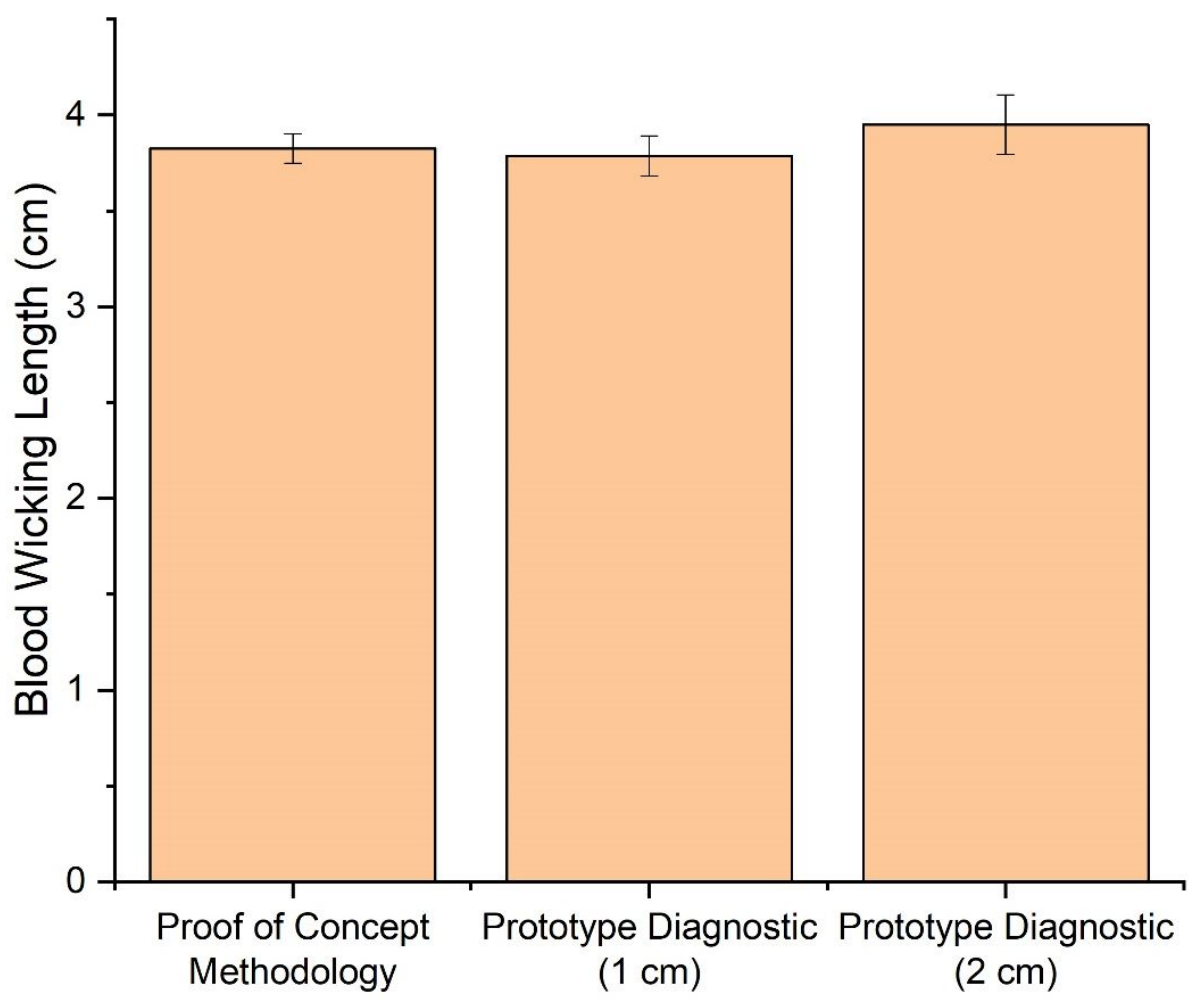

Supplementary Figure 16: Comparison of a whole blood sample (diluted 1:7) using the proof of concept methodology (as described in Section 3.6 and 3.7) and the prototype diagnostic (as described in Section 3.13). The paper strip in the diagnostic was released at variable heights (i.e. $1 \mathrm{~cm}$ and $2 \mathrm{~cm}$ ) above the glass slide. Each data point was tested 4 times and the mean and standard deviation are reported.

Supplementary Figure 16 compares the wicking and measurement of the prototype diagnostic at variable dropping heights to that of the proof of concept methodology. It determines the optimal dropping height of the diagnostic.

Regardless of the dropping height, the prototype diagnostic wicks very similarly to the proof of concept methodology (where the paper strip is manually released millimeters above the clotted droplet).

Although minor, the $2 \mathrm{~cm}$ dropping height causes the diagnostic to wick slightly further than the $1 \mathrm{~cm}$ dropping height. We hypothesise this to be due to the greater impact between the paper strip dropped at that height and the clotted droplet. Hence, Figure $\mathbf{1 0}$ displays the results of the prototype diagnostic with a $1 \mathrm{~cm}$ dropping height. 


\section{Wicking Front Enhancement}

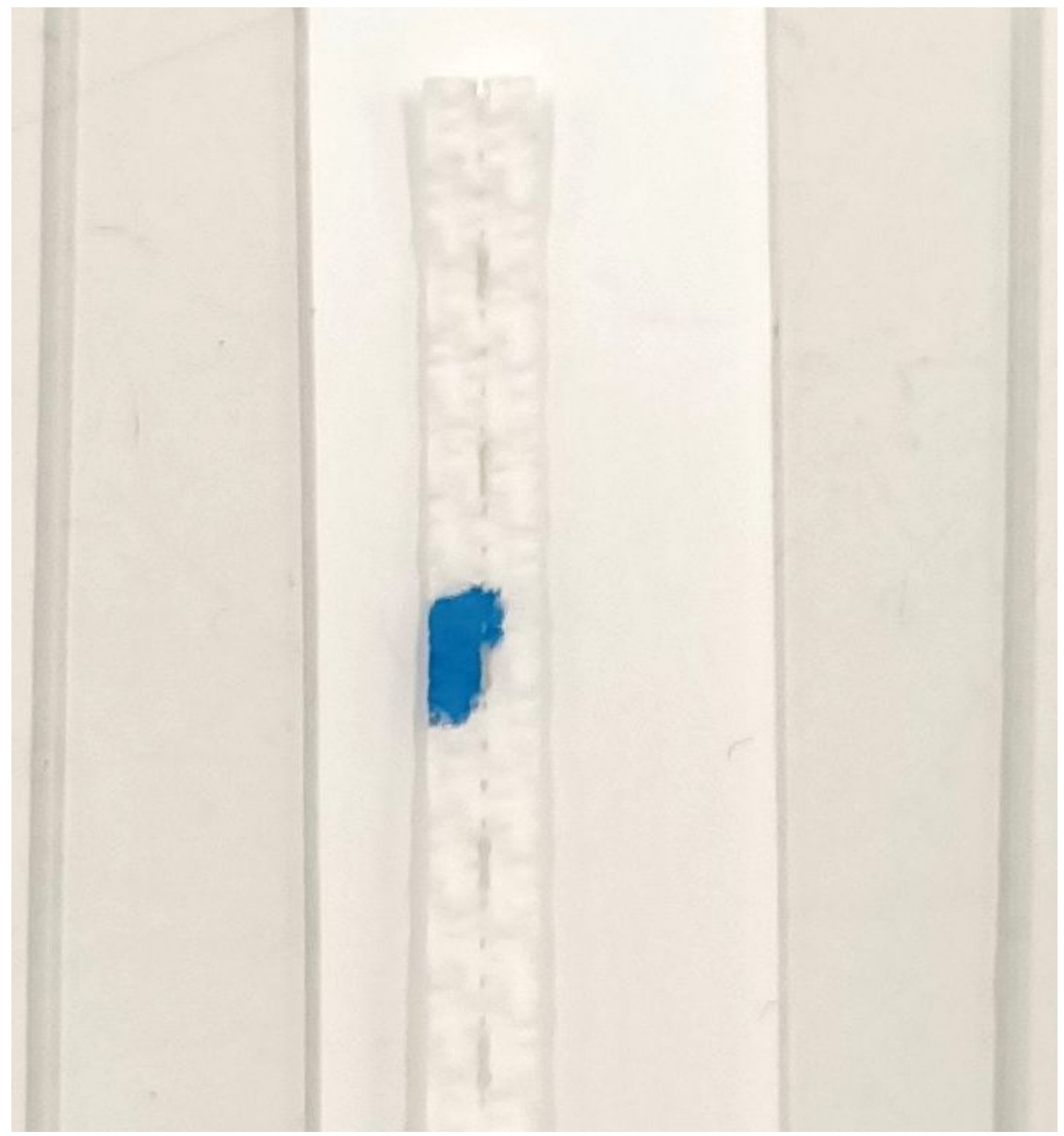

Supplementary Figure 17: Wicking front enhancement for diluted blood samples (ie. 1:3, 1:7 and 1:15). $0.1 \mu \mathrm{L}$ of Queens Blue Food Dye (undiluted) was pipetted $1.5 \mathrm{~cm}$ from the top of the paper strip.

Supplementary Figure 17 shows how wicking fronts from blood samples with poor visibility were enhanced. Diluted blood samples produced wicking fronts that were difficult to see and analyse. Hence, a minute amount of blue dye was added to the strip - downstream from where the paper strip landed on top of the blood sample.

After the paper strip was placed on the blood sample, the wicking front mixed with the blue dye so that it could be easily seen. 


\section{Terminology}

Supplementary Table I: Definition of common terms as used in the manuscript to provide clarity.

\begin{tabular}{|c|l|}
\hline Term & \multicolumn{1}{|c|}{ Description } \\
\hline Blood & $\begin{array}{l}\text { A sample/fluid composed of blood cells and/or components of } \\
\text { plasma of any composition. It is used in this manuscript as an } \\
\text { umbrella term (e.g. blood wicking length refers to the distance } \\
\text { that fluid from a blood sample has wicked down the paper strip). }\end{array}$ \\
\hline Whole Blood & $\begin{array}{l}\text { Blood sample which contains a mixture of blood cells and plasma } \\
\text { at physiologically relevant compositions (i.e. compositions that } \\
\text { are typically found in the blood stream). In this manuscript, a } \\
\text { whole blood sample refers to a pre-made mixture of 50\% red } \\
\text { blood cells and 50\% plasma (unless the composition is stated } \\
\text { otherwise). }\end{array}$ \\
\hline Unbeaded Droplet & $\begin{array}{l}\text { A mixture of thrombin solution and whole blood or plasma that is } \\
\text { immediately deposited on the PTFE-coated glass slide. }\end{array}$ \\
\hline Beaded Droplet & A droplet with an acute contact angle (<90 degrees). \\
\hline Clotted Droplet & $\begin{array}{l}\text { A droplet after the fibrinogen in it (if present) has polymerised } \\
\text { into fibrin and formed the fibrin scaffold. }\end{array}$ \\
\hline \multicolumn{2}{|c|}{ When } \\
\hline Clotted Phase & $\begin{array}{l}\text { The effect that RBCs and plasma proteins (other than fibrinogen) } \\
\text { have on blood wicking. As RBCs and plasma proteins both interact } \\
\text { with cellulose fibres and increase the viscosity of a blood sample, } \\
\text { they are able to slowdown the movement of blood through the } \\
\text { paper strips (irrespective of what the fibrinogen concentration of } \\
\text { the blood sample is). }\end{array}$ \\
\hline Effects & $\begin{array}{l}\text { The viscoelastic phase which consists of the fibrin scaffold and } \\
\text { entrapped RBCs. }\end{array}$ \\
\hline & $\begin{array}{l}\text { The liquid phase which consists of RBCs and unbound plasma } \\
\text { molecules that did not get entrapped by the fibrin scaffold. }\end{array}$ \\
\hline
\end{tabular}

